\title{
10 Notes au fil du texte
}

\section{Titre :}

Des offices : en moyen français, le mot office est un terme polysémique (ATILF, Dictionnaire du moyen français, s.v.). On peut le comprendre ici comme 'mission, tâche, rôle, service' envers qqn ou envers la société, ${ }^{1}$ sens attesté du mot français (Firmin Le Ver propose la glose «serviche» dans son dictionnaire latin-français). ${ }^{2}$ Il semble que pour Anjourrant le mot entrait dans la sphère des notions morales, comme le montrent son association avec vertu dans le prologue (0.16-17) et la traduction presque systématique d'officium par office et vertu, ${ }^{3}$ voire par vertu seul ; ${ }^{4}$ on pourrait souvent le gloser par le sens, également attesté, de 'devoir, obligation morale', 5 qui rend assez adéquatement la notion de kathekon que Cicéron cherche à traduire par le latin officium.

Cette vertu n'est pas uniquement lue dans une perspective morale et spirituelle, mais également pratique et politique. En effet, dans quelques cas, l'on peut penser que le traducteur convoque de façon concomittante le sens, également courant, de 'charge ou mission exercée dans le cadre du service de l'État'. ${ }^{6}$ Par exemple, dans les segments 17 et 18 du prologue, où le traducteur rapproche l'ordo médiéval et la res publica antique, Anjourrant souligne l'importance du texte de Cicéron et des vertus qu'il défend pour «ceulx qui par office ont le regime et administracion de la chose publique ». ${ }^{7}$ Sur ces questions, voir le chap. 3 de l'Introduction.

Cy commance ... : les témoins du groupe $\beta$ qualifient clairement le texte comme une œuvre didactique «qui traicte de justice et de injustice et des quatre

1 D'ailleurs, munus 'fonction, charge' est traduit par offices et vertuz (I.124.2).

2 Merrilees/Edwards 1994. Sur les sens du mot chez Cicéron, Testard 1984, t. I, $54-56$; Hellegouarc'h 1963, 152-163 ; Dyck 1996, 13-24.

3 «car ta vie ne peult estre sans les offices et vertus» (I.4.6); «aucunes disciplines des offices et vertus» (I.5.2) ; «ilz ne pourroient dire aucune chose de office et vertu» (I.6.2) ; «le droict office ou vertu» (I.8.2) ; voir aussi I.19.4 ; I.30.5 ; I.31.5 ; I.32.8 ; I.47.4 ; I.100.1 ; I.103.1 ; I.107.3 ; I.160.1. La traduction du mot officium par office en emploi autonome est rare (I.59.3, I.61.1, III.14.1).

4 I.122.1, I.147.2, I.152.1.

5 David Miffant choisira également office pour traduire officium dans sa traduction tandis qu'en 1547, Louis Meigret utilisera le plus monosémique devoir, choix des traducteurs jusqu'à aujourd'hui.

6 Sur la définition médiévale d'office, voir Autrand 1969 ; Lachaud 2010, 175-220.

7 Sur cette utilisation du mot officium dans la sphère politique, voir Lachaud 2009 ; Brunt 1986, 12-32.

○ Open Access. () 2019 Olivier Delsaux, published by De Gruyter. (c) BY-NC-ND This work is licensed under the Creative Commons Attribution-NonCommercial-NoDerivatives 4.0 License.

https://doi.org/10.1515/9783110621365-010 
vertus cardinalles » (cf. la miniature d'ouverture du manuscrit $\mathrm{V}_{1}$ ), suivant là les propos du traducteur dans son prologue. Celui-ci identifie les quatre vertus cardinales (sapience, justice, force et actrampance) comme une voie nécessaire à l'acquisition de la sapience generale (0.8). Cette perspective correspond à la réception courante du De officiis au Moyen Âge (cf. Introduction, section 3.1).

0.1-07 Pour ce que l'umain lignage ... : nous n'avons pas pu identifier avec précision la ou les sources du traducteur pour ces développements très généraux sur l'importance de la sagesse comme moyen d'accès à la beatitude ; ils rappellent certains passages du Livre vil du Policraticus de Jean de Salisbury (voir éd. Brucker 2013, en part. chap. 13 à 15 ; voir également Introduction, section 3.1).

0.8 qui n'est pas prise si generallement ... : idée développée aux segments I.17I.19 du texte de Cicéron.

0.10 Cellui qui a la vertu de justice a toutes les vertus : citation non identifiée avec certitude ; il pourrait s'agir d'une phrase attribuée à saint Jérôme, présente dans la compilation de sentences morales qu'est le Manipulus florum (s.v. Iusticia et iustus ${ }^{8}$ ) : "Omnes uirtutum species uno iusticie nomine continentur. Ieronymus ad Demetriadem » [Pélage, Epistola ad Demetriadem, 5, PL 30, col. 21a].

0.11 Et, à ce propos, dit le philozophe ... : Arist. Pol. I.2, très certainement dans la traduction de Guillaume de Morbeke. ${ }^{9}$

0.12 Ores, plusseurs philozophes et sages gens ... : l'augustin Jacques Legrand avait rédigé, à la toute fin du XIV siècle, le Sophilogium (partiellement auto-traduit dans son Archiloge Sophie, très bien diffusé au XV siècle), qui traitait précisément de l'amour de la sagesse et des sciences, d'une part, et de l'amour des vertus théologales, cardinales et capitales, d'autre part. ${ }^{10}$ Plus globalement, il pourrait être fait référence au Livre de la Sagesse, notamment au verset : Sobrietatem enim et prudentiam docet et iusticiam et uirtutem quibus utilius nichil est in vita hominibus. (8.7.)

0.13 ilz n'entendent pas ... : l'émendation de la leçon de R se justifie par l'accord des deux autres familles contre elle ; notre choix de la leçon de $\alpha$ s'explique par le fait que la leçon de $y$ (point) correspond à une modification linguistique (point pour pas) que l'on observe fréquemment dans $\gamma$.

8 Édition électronique par Chr. L. Nigham, Waterloo University, http://web.wlu.ca/history/ cnighman/ (consulté le $1^{\text {er }}$ mai 2018).

9 Éd. Susemihl 1872, 10 (Bekker 1253a).

10 Beltran 1986. 
0.13 pour ce que aucunes gens ... : justification courante de la traduction : l'accès des moins lettrés au savoir des lettrés et le risque de mauvaise interprétation des textes écrits. ${ }^{11}$

0.14 aucun petit livret : il s'agit vraisemblablement du Breuiloquium de uirtutibus antiquorum principum atque philosophorum - autrement connu sous le nom de Libellus de quatuor uirtutibus cardinalibus / Liber quatuor uirtutum / Breuiloquium de quatuor uirtutibus cardinalibus - de Jean de Galles. ${ }^{12}$

0.15 Tanguy du Chastel, seigneur de Regnac : sur sa biographie, voir chapitre 1.

0.21 Car, comme dit le sage ... : Vulgate, Sap. 3.3.

0.22 Et combien que je cognoisse ... : Anjourrant applique un conseil énoncé par Cicéron dans le texte (I.147.1).

0.23 Et, pour tant, seigneurs qui vouldrez passer le temps ... : le motif de la commande d'une traduction par passe temps est assez récent $;^{13}$ les prologues des traductions de textes didactiques antérieures mettent plutôt en avant l'utilité de la traduction. ${ }^{14}$

0.24 sans y comprendre aultre chose que le sens licteral ... : la fidélité au sens littéral est un topos de nombreuses traductions françaises de la fin du Moyen Âge. ${ }^{15}$

I.14.3 comme en faiz : émendation fondée sur l'usus scribendi : "tant en diz comme en faiz» (I.103.3) ; "tant en chevauchant et en gectant dars comme en souffrant toutes choses par labour de chevallerie» (II.45.8) ; «tant en plaidoiant en jugement come en deliberant au senat » (II.49.1).

I.15.2 comme dit Platon : ut ait (leçon-source de ce lieu du texte) est traduit par comme recite en II.41 et par comme dit en III.61.

11 Voir, par exemple, Lusignan 1986 ; Lusignan 1989 ; Boucher 2005 ; voir aussi Introduction, section 3.1.

12 Hauréau 1868 ; Mombello 1967, 13-15 ; Bloomfield 1979, 422-423 ; Boureau 2014, 153-171. Le texte est toujours inédit ; nous avons utilisé l'édition publiée à Lyon en 1511 (Summa Joannis Valensis de regimine vite humane).

13 Voir, par exemple, le prologue de la pseudo-traduction que sont les Chroniques de Pise (c. 1470) : «Pour tant que parolles sont vaines et escriptures permanentes, pensoie, n'a pas long temps, que pour rendre aulcune petite oeuvre à cellui mon treschier et redoubté seigneur qui moult voulentiers se occupe et passe temps à oyr lire et retenir les fais des anchiens, et sans plus exillier le temps advenir, car du passé trop en ay perdu en vain et sans labeur qui à moy chetif est chose inrecouvrable» (Bruxelles, KBR, 9209, fol. 1r); voir aussi : «Et ce ay je fait affin de donner sollacieux plaisir et passe temps à vostre esperit travaillé et fatigué de si grandes et intollerables paines par vous passees et domptees.» (Octovien de Saint-Gelais, traduction du De Christiane fidei et Romanorum pontificum persecutionibus de Boniface Symonetta, Paris, A. Vérard, [c. 1507], exemplaire Paris, Bibl. de l’Arsenal, $4^{\circ} \mathrm{H} 6425$, fol. 1r). Sur cette question, voir aussi Cerquiglini-Toulet 1995.

14 Monfrin 1972.

15 Buridant 2011. 
I.20.5 Par ire : variante du manuscrit A : " par yre qui est ung vice qui contrarie moult fort la voye de verité».

I.21.4 Alixandre ... : la brève référence à Alexandre le Grand insérée au milieu du raisonnement de Cicéron au I.21.3 est bien une figure exemplaire ajoutée par le traducteur - au même titre que la large addition sur Golias et Saül [Samuel, I.17] qui suit (I.21.6) - ; d'où le fait que le manuscrit R fasse commencer l'addition du traducteur avec Alexandre. Cependant, le segment qui suit immédiatement (I.21.4-5) est bien encore le texte de Cicéron. La leçon de $\mathrm{R}$ pourrait s'expliquer par une anticipation par le copiste de «comme nous lisons » (I.21.6) au moment de transcrire «ainsi que» (I.21.4) ou par de l'existence de leçons concurrrentes dans l'archétype pour l'introduction de l'exemplum d'Alexandre (par exemple un ajout en marge avec des signes de renvoi susceptibles d'interprétations diverses). L'accord de $\gamma$ et $\alpha$ contre la leçon de R nous oblige à corriger sa leçon.

I.21.6-10 Comme nous lisons ... : source générale : Sam I.17.

I.26.5-6 nous avons que Nabugodenosor : source générale : Dan 4.

I.26.7-8 Pareillement, nous trouvons de David ... : source générale : Sam II.24 et Chroniques I.21.

I.32.1-5 car, comme nous trouvons es fables ... : le développement de la fin tragique d'Hippolyte, fils de Theseus, pourrait provenir des Métamorphoses d’Ovide (Livre XV, v. 479-551, éd. Lafaye 1960), peut-être en suivant les développements de l'Ovide moralisé. ${ }^{16}$

I.33.5 comme celui qui fist triefves : il pourrait s'agir de Cléomène (cf. éd. Testard, I, p. 194) ; Anjourrant ne semble avoir fait que développer des éléments implicites du texte.

I.33.10-11 Et, quant Fabius Labeo ... : amplification des éléments implicites du texte ; pour cet épisode, Anjourrant a pu s'appuyer sur Valère Maxime (Facta et dicta memorabilia 7.3.4).

I.37.4-5 Et, pour ce, se tu me demandes ... : le texte-source a : Equidem etiam illud animaduerto, quod, qui proprio nomine perduellis esset, is hostis uocaretur, lenitate uerbi rei tristitiam mitigatam. Hostis enim apud maiores nostros is dicebatur, quem nunc peregrinum dicimus. Le traducteur n'a pas parfaitement saisi le sens du mot perduellis (Firmin Le Ver le glose par champion) et la traduction de peregrinum par pelerin rend difficile la compréhension $\mathrm{du}$ lien entre le sens primitif d'hostis 'étranger' et peregrinus.

I.38.8-11 Et en la bataille ... : les développements du traducteur relatifs à Regulus proviennent sans doute des propres développements de Cicéron au Livre III (III.99-115).

16 De Boer 1938, vv. 1229-1452. 
I.40-41 Le paragraphe 40 n'est pas traduit. Le fait que la fin du paragraphe 38 et le paragraphe 39 soient traduits de façon extrêmement lâche, omettant plusieurs éléments du texte-source, pourrait laisser penser que le traducteur disposait d'un modèle lacunaire à cet endroit et qu'il a tâché de broder autour de l'histoire de Regulus.

I.53.1 affin que nous nous departons : il faut sans doute lire departions (leçon de L) : affin que se construit majoritairement avec le subjonctif dans le textecible et le texte-source donne discedatur.

I.61.9 et sont mises es coings des rues ... : source non identifiée pour cette addition relative à l'usage de statues figurant les militaires armés ; il pourrait s’agir d'une simple amplification de l'idée exprimée par Cicéron.

I.67.2 si est pour ce que ... : émendation justifiée par l'accord des deux autres familles contre hardement, qui serait la seule attestation du texte (cf. Glossaire).

I.68.8 ne les doivent point prendre : ne doivent + infinitif se construit généralement avec le renforcement pas ou point (voir par exemple I.28.5, I.38.2, I.49.9, I.87.4, I.125.1, I.128.1, II.29.5, II.77.12, etc.) ; d'où notre émendation de la leçon de R.

I.71.9 et sont meuz : seule la forme sans $l$ est attestée ailleurs dans le texte : esmeuz (I.73.3, I.102.7, II.2.5, II.22.4, II.32.2).

I.75.1 Themistodes : il s'agit bien de Thémistocle, mais la leçon de L (Themistocles) est certainement une émendation de la leçon de l'archétype ; l'on observe plusieurs cas où l'imprimé L propose une forme de nom propre plus proche du texte-source.

I.76.3-23 Car, ainsi que recite Tregus Pompeius en son tiers livre ... : cette addition provient de Justin, très certainement par l'intermédiaire du Breuiloquium de Jean de Galles : ${ }^{17}$ Unde narrat Trogus Pompeius liber III de Ligurgo, que cum statuisset mandata et leges quod dura Lacedemoniis uidebantur, auctorem earum Apollilinem Delphicum finxit, et se simulans proficisci ad oraculum Delphicum obligauit iuramento ciuitatem ut nihil in legibus illis mutarent antequam ipse reuerretur. Quo facto fuit ad Cretam ibique perpetuum elegit exilium, ut dicte leges obseruarentur. Moriens uero ossa sua abiici in mare precepit, ne si reportarent, ciues soluerent iuramentum, estimantes se ab illo solutos. [...] Et quod dicte leges erant honeste hic suscribantur :

[1] Prima lege : Populum in obsequium principum et principes ad eorum iustitiam informauit ;

17 L'attribution à Trogue Pompée n'apparaît que dans le Breuiloquium, qui introduit les douze lois en des termes proches de la conclusion de l'addition d'Anjourrant ; les variantes par rapport à Justin confirment l'emploi de cet intermédiaire. 
[2] Secunda lege : Parsimonia omnibus suasit, estimans laborem militiae assidua frugalitatis consuetudine faciliorem fore ;

[3] Tertia lege : Emi singula non pecunia, sed mercium conpensatione iussit ;

[4] Quarta lege : Auri usum uelut omnium scelerum mater sustulit ;

[5] Quinta lege : Administrationem rei publice per ordinem diuisit. Regibus potestatem bellorum, magistratibus iudicia et annuas sanctiones. Senatui custodiam legum, populo eligendi et creandi quos uellet magistratus permisit ;

[6] Sexta lege : Omnes fundos inter equaliter diuisit, equa patrimonia neminem aliis potentiorem redderent ;

[7] Septima lege : Conuiuari omnes publice iussit, ne cuiusque delitie uel luxuria in occulto essent ;

[8] Octava lege : Iuuenibus non amplius una ueste in toto anno uti permisit ;

[9] Nona lege : Pueros puberes non in forum, sed in agrum deduci iubet, ut primos annos non in luxuria, sed in opere agerent ;

[10] Decima lege : Statuit uirgines nubere sine dote ;

[11] Undecima lege : Iussit ut uxores legerentur non pecunia ;

[12] Duodecima lege : Maximum honorem non diuitum uel potentium, sed pro gradu aetatis senum esse uoluit. (Breuiloquium, col. ccb, qui suit Just., Epit., III, 2-3, éd. Seel 1972).

I.84.9 Epannimunde : Épaminondas de Thèbes, identifié comme « Thebane et Epaminunde» au I.155.5.

I.87.1 Contenement : le mot signifie 'comportement, manière de se comporter, allure' et ne fonctionne donc pas parfaitement comme équivalent de contentio (Le Ver : contencion, noise, estris ${ }^{18}$ ). Vu que le verbe régi par la coordination ambicion et contenement est conjugué au singulier, l'on peut penser que le traducteur estimait ambicion et contenement sémantiquement proches. Le leçon contenement pourrait être une erreur de l'archétype pour contendement 'aspiration, ambition'.

I.94.1 ce qui est honneste est honnourable ... : Anjourrant rend la notion antique de decor 'convenance' par honneur (Le Ver propose biauté, mais précise également que le mot était un équivalent d'honor pour les «antiqui ») $;^{19}$ ce choix semble délibéré dans la mesure où le traducteur traduit deceat par est convenable au I.97.5.

I.96.4-7 Mais, se celles diffinicions ... : Est igitur decorum quod praeeminet, cuius diuisio gemina est. Nam est decorum quasi generale quod per uniuersitatem funditur honestatis et quasi in toto spectatur corpore ; est etiam speciale quod in parte aliqua enitet. [...] Habes hunc decorem generalem

18 Merrilees/Edwards 1994, s.v.

19 Merrilees/Edwards 1994, s.v. 
quia fecit Deus mundi istius pulchritudinem. Habes et per partes quia cum faceret Deus lucem, et diem noctemque distingueret, cum conderet caelum, cum terras et maria separaret, cum solem et lunam et stellas constitueret lucere super terram, probauit singula. (saint Ambroise, De officiis, I, 46, éd. Testard 2000, 82).

I.101.5 Car, comme dit Saluste : sed nostra omnis uis in animo et corpore sita est : animi imperio, corporis seruitio magis utimur (Sall. Cat. 1.2, éd. Kurfess 1957, 2).

I.102.9 car l'esguillon de ire ... : pourrait suivre Grégoire le Grand : Nam irae suae stimulis accensum cor palpitat, corpus tremit, lingua se praepedit, facies ignescit, exasperantur oculi et nequaquam recognoscuntur noti. (Moralia in Job V.45, éd. Adriaen 1979-1985, cité dans le Manipulum florum, s. v. «ira»).

I.103.1 affin que nous retournons : il faut sans doute lire retournions; affin que se construit avec le subjonctif ailleurs (cf. "Remarques linguistiques ») ; le texte-source, dont Anjourrant suit souvent fidèlement la concordance des temps, a reuertamur.

I.105.6-7 Et, ainsi que dit Saluste ... : Omnes homines, qui sese student praestare ceteris animalibus, summa ope niti decet, ne uitam silentio transeant ueluti pecora, quae natura prona atque uentri oboedientia finxit. (Sall. Cat. 1.1).

I.109.9 comunité de langage : la leçon comité de L est morphologiquement plus proche du latin comitatem. Cependant, la leçon commune commun en langaige à la phrase suivante confirme que le traducteur a sans doute lu comunitatem ou a traduit comitatem par un terme formellement proche. Le confirme également la traduction de comitas par compagnie au II.48.5.

I.111.2 Rien dans le texte n'indique que l'on oppose ici les hommes aux bêtes.

I.112.2-4 Car, nous avons vëu que quant Cesar ... : chez Cicéron, le passage oppose Jules César et Caton d'Utique. ${ }^{20}$ Cependant, les additions du traducteur proviennent, semble-t-il, plutôt de la vie de l'empereur Auguste, qui aurait été refusé par le Sénat en raison de son âge («les Romains ne le vouloient pas pour ce qu'il estoit trop jeune») : au mois d'août 43, il fit sa marche sur Rome et le 19 fut élu consul par la force («il vint devant Rome à tout son armee et entra dedans») ; la précision «des jeunes gens les plus mauvais qu'il peust trouver» pourrait se référer au cousin d'Auguste, Quintus Pedius [RE, $n^{\circ} 1$ / MRR, 2.599]. Par contre, la mention de l'Afrique ne fait pas sens et témoigne de la confusion avec Jules César. ${ }^{21}$ Resterait à trouver la source éventuelle qui a conduit Anjourrant à cette confusion. L'on notera qu'Auguste est fréquemment identifié comme Cesar ou Caius/Gayus, notam-

20 Dyck 1996, 282.

21 Cette lecture nous a été aimablement suggérée par le Professeur Ermanno Malaspina. 
ment chez un traducteur français contemporain d’Anjourrant, Sébastien Mamerot. 22

I.121.7-14 Nonobstant que Mycipsa : source générale, résumée : Sall. Iug. 5, 6, 11, identification proposée par Bossuat 1935, 269.

I.130.5-6 car, quant ilz veullent exprimer ... : sur cette question, voir quelques pistes de réflexion dans Koopmans 1997.

I.137.7 avoit de coustume : la structure partagée par R et L (avoir de coustume de) est bien attestée dans le texte (I.129.5, I.129.7, III.33.3, III.80.3).

comme recite Therence ... : il s’agit en réalité de Pyrgopolynice au centre du Miles gloriosus et non de Traso dans l'Eunuchus.

I.138.4-7 Scaurus : Marcus Aemilius Scaurus n'est pas le fils de Gnaeus Octavius ; le texte précise qu'il fut le filius d'un summus et clarissimus uir, d'où la confusion d'Anjourrant.

I.139.8 Odiosum est ... : il s'agit du dernier lieu du texte où le manuscrit $\mathrm{V}_{1}$ propose un extrait du texte latin.

I.140.6 en toutes ces choses : la leçon unique de $\mathrm{R}$ a été émendée ; seule la séquence en toutes choses est attestée dans le texte (I.73.7, I.86.3, I.133.12).

I.140.6 celle mesme mediocrité : la leçon séparative de $\mathrm{R}$ est maintenue : la leçon de $\gamma$ et de $\alpha$ pourrait être une faute polygénétique assez facile (simplification des initiales me-).

I.148.4-5 Semblablement, pouons nous dire de Pithagoras ... : source non identifiée ; pourrait s'inspirer de Valère Maxime (Facta et dicta memorabilia, 8.15.1).

I.148.6-8 Pareillement, Platon fut si eloquent ... : l'épisode est relaté dans la Vie de Platon d'Olympiodore (VI ${ }^{\mathrm{e}} \mathrm{s}$.), qui se présente au début de son commentaire de l'Alcibiade. ${ }^{23}$ Reste à identifier l'intermédiaire qu'a utilisé Anjourrant ; il pourrait s'agir de Valère Maxime (1.6.ext.3).

I.151.6-10 Ou quel livre ... : Venio nunc ad uoluptates agricolarum, quibus ego incredibiliter delector; quae nec ulla impediuntur senectute et mihi ad sapientis uitam proxime uidentur accedere. (Cic. Sen. 51, éd. Wuilleumier 1940) [...] Num igitur horum senectus miserabilis fuit qui se agri cultione oblectabant ? Mea quidem sententia haud scio an nulla beatior possit esse, neque solum officio, quod hominum generi uniuerso cultura agrorum est salutaris, sed et delectatione qua dixi et saturitate copiaque rerum omnium, quae ad uictum hominum, ad cultum etiam deorum pertinent ut, quoniam haec quidam desiderant, in gratiam iam cum uoluptate redeamus ; semper enim boni

22 Duval 2001, 308. Sur la figure de César dans la littérature française du Moyen Âge et de la Renaissance, voir Méniel/Ribémont 2006-2007.

23 Westerink/Trouillard 1990, Prolégomènes, II, 15-30, p. 4. 
assiduique domini referta cella uinaria, olearia, etiam penaria est uillaque tota locuples est, abundat porco haedo agno gallina lacte caseo melle; iam hortum ipsi agricolae succidiam alteram appellant. [...] agro bene culto nihil potest esse nec usu uberius nec specie ornatius » (ibidem, 56-57). Anjourrant ne suit pas la traduction de Laurent de Premierfait.

I.151.11-12 Car, ainsi que nous recite Ysodore : Cultura est qua frumenta uel uina magno labore quaeruntur, ab incolendo uocata. Diuitiae enim antiquorum in iis duobus erant, bene pascere et bene arare (Isidore de Séville, Etymologiae, 17.2.1, éd. Lindsay 1911).

I.157.7-19 Et, à ce propos, dit Gallensis ... : Remota itaque justitia, quid sunt regna, nisi magna latrocinia ? [...] Nam cum idem rex hominem interrogaret, quid ei uideretur, ut mare haberet infestum, ille libera contumacia : 'Quod tibi, inquit, ut orbem terrarum; sed quia id ego exiguo nauigio facio, latro uocor ; quia tu magna classe, imperator'. " (saint Augustin, Cité de Dieu 4.4, éd. Dombart/Kalb 1981) ; le traducteur, comme il l'avoue lui-même, suit le Breuiloquium de Jean de Galles, en particulier pour les détails, notamment le nom du pirate : "Quia latrocinia sunt parua regna ait Augustinus III De cita Dei, IIII. Introducens exemplum de Alexandro et pirata, de quo exemplo planius et plenius narratur lib. III « de nugis philosophorum », ubi dicitur quod cum pirata esset deprehensus et Alexander interrogaret, propter quod haberet mare infestum, respondit : Libera contumacia, propter quod tu orbem terrarum, sed quia id ego facio uno nauigio, "latro" uocor ; quia tu magna classe facis, diceris «imperator». Si solus captus fit Alexander latro erit. Si ad nutum Dionidis populi famulentur, erit Dionides imperator. Vocabatur enim Pirrata Dionides. Nam quam tum ad causam non differunt, nisi quod deterior est qui rapit improbius, quam qui iusticiam abiectius deserit, quia manifestius impugnat. Leges quas ego fugio, tu persequeris. Ego eas uicumque ueneror, tu contemnis. Me fortune iniquitas et rei familiaris angustia. Te fastus intollerabilis et inexplebilis cupiditas furem facit. Si Fortuna mansuesceret fierem forte melior. At tu quo fortunatior nequior eris. Miratus Alexander constantiam hominis eum merito arguentis [?] : experiar inquit an futurus fis melior. Fortunam mutabo, ut non ei amo quid deliqueris, sed tuis moribus ascribatur. Eunque fecit, militie ascribi, ut posse exinde saluis legibus militare. » (col. CCI a). ${ }^{24}$

II.2.5 Je ne me adonnay point à tristesse ... : seul le manuscrit $\mathrm{V}_{1}$ (fol. 44v) met en évidence cette sentence au moyen de l'annotation marginale de l'équivalent latin. L'annotation est de la main du copiste et contemporaine de la transcription du texte. Vu que depuis le fol. 38r (I.138), ce manuscrit ne présente

24 Sur cette idée, voir aussi Sen. ep. 30-32 ; voir Malaspina 2018. 
plus aucune des mises en évidence des extraits du texte-source latin que l'on trouve dans les autres manuscrits, il n'est pas à exclure qu'il s'agisse d'une intervention extérieure au texte de l'archétype.

II.5.3 Elle doit estre mise ... : émendation sur la base d'un passage parallèle : «elle doit estre mise devant sapience» (I.153.11).

II.5.4-6 Et, à ce propos, nous avons que ... : Anjourrant a pu suivre le Breuiloquium : Item quam fortes fuerunt in sustinentia ablationis rerum suarum praeter ex dictis. Unde narrat Valerius liber VII [Val. Max. 7.2.2] de quodam philosopho qui dictus est Bias Priennum, quod cum hostes patriam suam Prienen inuasissent et homines fugerent cum rebus suis interrogatus ille respondit : Omnia mea mecum porto. Pectore enim gestabat, non humeris, neque oculis uisenda sed animo estimanda, que domicilio mentis inclusa neque mortalium, neque deorum manibus labefactari possunt et ut manentibus presto sunt, ita fugientibus non desunt. (Jean de Gales, Breuiloquium, fol. ccxvi b).

II.5.7-11 Semblablement, nous raconte Seneque ... : Consimiliter ait Seneca libro «De constantia sapientis" [Sen., Constant. 5.6, éd. Hermes 1923, 27] quod cum Democritus Mageram cepisset. Stipho philosophus ab eo interrogatus si aliquid perdidisset : Nihil, inquit, omnia mea mecum sunt. Et tamen patrimonium et filios hostes rapuerant [sic] qui inuictum et indemnem se testatus est. Habebat enim uera bona in quo non est manus injectio. Et dissipata ac dirrupta, ideo non ut sua dilexerat. Nec talia bona esse reputabat philosophi. (Jean de Gales, Breuiloquium, fol. ccxi a).

II.16.2 Themistodes, Perules, Sirus, Angesilaus : nous n'avons pas émendé la leçon de $\mathrm{R}$, partagée par $\mathrm{V}_{1}$ et $\mathrm{P}$; la leçon de $\mathrm{L}$, certes plus proche de l'exactitude grammaticale, nous semble une modification non auctoriale, sans doute due à une émendation lors de la composition du texte pour les presses.

II.18.4 Nous puissons avoir : il faudrait peut-être lire puissions ; dans le texte, affin que se construit avec le subjonctif et le latin a habeamus.

II.18.7-10 Et, pour monstrer que ... : Anjourrant cite sans doute à nouveau le Breuiloquium : Ibidem ait ["Valerius ab vi»= Val. Max. 6.5, 1d] quod Timocrates pollicitus est se Pirrum ueneno necaturum. Que res cum ad senatum esset delata, missis legatis Pirrum monuit, ut aduersus insidias huiusmodi cautius se gereret. Maluit enim bella non ueneno sed armis gerere. Nomen quoque Thimocratis suppressit utroque modo amplexus equitatem, quia neque hostem malo paratus fuerat prodere noluit. (col. ccIII a).

II.18.7 nous ne devons point vanger ... : la leçon de la uaria lectio (construction pronominale du verbe venger) est défendable : la construction se vanger est attestée en I.34.2.

II.23.6-20 Et, à ce propos, nous recite Macrobe : Anjourrant suit le Breuiloquium, plus complet que le texte de Macrobe : Ait Tul. I Tusq. [Cic. Tusc. 5.21.61, éd. 
Pohlenz 1918] De Dionysio Tyranno, quod cum quidam amicus eius Damacles nomine laudaret copias eius et opes manifestatem damnatus rerum humanarum, magnificentiam edium regiarum, negaretque unquam quemquam beatiorem fuisse respondit. Vis degustari et experiri fortunam meam. Cumque se id uelle diceret : collocari enim fecit Dionysius in aureo lecto pulcherrimo et mensam parari opulentam, pueros et iuuenes ad ministrandum electos consistere fecit. Cum autem omnes adessent delitie. Et Damocles : sibi uideretur fortunatus, iussit tyrannus fulgentem gladium elimatum sera equina appensum demiti, ut penderet capiti illius et ceruicibus. Cumque ille : nec pulchros ministros aspiceret, nec manum ad mensam porrigeret, nec in lectum aureum pro timore quiesceret ne gladius super caput eius cadere ait tyrannus. Talis est uita mea, quam beatam putas. Ille uero orauit tyrannorum [sic] ut abire liceret. Vnde satis uidetur Dionysus declarasse : nullum beatum esse cui aliquis timor semper impendeat. Et de hoc eodem Macrobius lib. I [Macrob. In Somn. 1.10.16, éd. Willis 1970, 44] (fol. cCVII d).

II.23.16 s'il se demenoit : demener 'bouger, agiter' non pronominal est attesté, mais il est alors transitif (DMF, s. v. «démener »).

II.24.8 qui se veulent faire craindre : l'usus scribendi confirme la leçon de R : «qui les face plustost estranger que se faire craindre» (II.23.1) ; «quant il se voulut tant faire craindre» (II.25.1) ; «pour ce qu'ilz se vouloient faire craindre» (II.26.5)

II.29.1 comme fist Lucius Scilla : bien qu'homonyme du célèbre Scilla nommé au II.27 (Publicus Cornelius Sulla Felix), il s'agit d'un autre individu (Publius Cornelius Scilla), parent de celui-ci ; cf. Index des noms de personnes et de lieux.

II.34.3-18 Et, à ce propos, nous recite Helinandus ... : Ulterius de affectu eorum et humiliter etiam erga inferiores luculenter exprimitur in gestis eorum. Unde Helynandus in "Gestis Romanorum " narrat de Trayano qui cum equum ascendisset ad bellum profecturus, uidua quedam apprehenso pede eius, miserabiliter lugens, iusticiam sibi petiiit de eis, qui filium eius bonum et innocentem occiderant. Dixit : Tu, Auguste, imperas, et ego tam atrocem iniuriam patior? Cui imperator : Ego tibi satisfaciam cum rediero. Et illa : Quid si non redieris? Successor, inquit, meus satisfaciet. At illa : Quid tibi prodest, si alius benefecerit? Tu mihi debitor es, secundum merita recepturus. Et fraus utique est, nolle reddere quod debetur. Successor tuus iniuriam pro se patientibus tenebitur, te quoque non liberabit iusticia aliena. Bene enim agetur cum successore tuo si se liberauerit. His uerbis motus imperator descendit de equo et causam examinans presentialiter, condigna satisfactione uiduam consolatus est. Unde et in foro statua Trayani posita est representans quando in expeditione positus, uiduam liberauit et in senatu exclamatum fuit. Non alter feli- 
tior Augusto nec melior Trayano. (Jean de Gales, Breuiloquium, col. ccirI b [suit sans doute le Speculum historiale de Vincent de Beauvais, 9.46 (De imperio Traiani), qui propose également la référence à la Chronique universelle d'Hélinand ; texte du manuscrit Douai, BM, 797, mis en ligne par I. Draelents, Base Speculum historiale (Nancy, Atilf) ; Anjourrant ne suit pas la traduction de Jean de Vignay (Paris, BnF, fr. 314, fol. 126v)]).

II.34.18-19 Et, pour tant, justice conjoincte à prudence ... : les phrases 18 et 19 pourraient toutes deux être la traduction du texte-source iustitia sine prudentia multum poterit. S’agit-il d'une trace de deux leçons concurrentes remontant au manuscrit du traducteur?

II.38.6-8 ne leur pouoir commander : Consimile narrat Vegetius, lib. IIII, "De re militari » [en réalité Val. Max. 4.3.6], quod cum legatus Epirotharum Fabricio consuli grande pondus auri offerret illo non accepto, dixit : se male illud habentibus imperare quam illud habere. (Breuiloquium, col. ccII c).

II.41.1 pour cause de user de justice : la construction "pour cause de » (leçon de $\mathrm{R})$ est attestée, mais pas "pour la cause de» (leçon de $\alpha$ ).

II.42.4-6 Et les loys sont faictes ... : source possible : Factae sunt autem leges ut earum metu humana coherceatur audacia, tutaque sit inter inprobos innocentia, et in ipsis inprobis formidato supplicio refrenetur nocendi facultas. Legis enim praemio aut poena uita moderatur humana. Erit autem lex honesta, iusta, possibilis, secundum naturam, secundum consuetudinem patriae, loco temporique conueniens, necessaria, utilis, manifesta quoque, ne aliquid per obscuritatem in captionem contineat, nullo priuato commodo, sed pro communi ciuium utilitate conscripta (Isidore de Séville, Etymologiae $2.10 \mathrm{De}$ lege).

II.42.8 leur empereur ou pour leur roy : émendation du ms. R justifiée par un souci de cohérence avec la phrase précédente (II.48.7), où il est déjà question, dans $\mathrm{R}$ et $\alpha$, d' «eslire [...] leurs empereurs ou roys» (II.48.7).

II.43.7 ont mis toute leur gloire en faintise : seule la construction de faintise avec la préposition en est attestée : "se Ulixes eust perseveré en celle faintise» (III.98.1).

II.48.2-3 comme nous avons dit en nostre premier livre ... : pour son développement, Anjourrant s’appuie sur le paragraphe I.132 du texte-source.

II.55.4 car pour quoy, quel fons ... : le passage semble corrompu ; le latin a : Etenim quis potest modus esse, cum et idem, qui consueuerunt et idem illud alii desiderent.

II.61.1 et descent de la vertus de l'omme ... : rappel des Offices II.52.

II.71.10-12 Et, à ce propos, nous recite Jerosme ... : De continentia aliorum philosophorum ait Hiero. epistola XXXV dixit quod Socrates Thebanus homo quondam ditissimus cum ad philosophandum Athenas pergeret, magnum auri pon- 
dus abiecit, neque putauit se posse uirtutem simul et diuitias possidere. (Jean de Gales, Breuiloquium, fol. ccxI a) ; l'anecdote, y compris la référence à saint Jérôme, se trouve également chez Thomas d'Aquin (Contra impugnantes Dei cultum et religionem, 3.3, nº 220, éd. Leonina, t. XLI A, 1970).

II.71.13-21 Semblablement, nous recite Valere en son quart livre ... : Currius consul cum Samnites magnum sibi pondus auri attulissent. Quibus ille ait : Non aurum habere, preclarum sibi uideri, sed eis qui aurum habent potius donari. (Breuiloquium, col. ccII c) [...] Narrat etiam ibi Valerius [Val. Max. 4.3.5, qui développe davantage] Ma. Curio et eius continentia, quod fuit norma Romane frugalitatis et specimen fortitudinis, ad quem cum legati Samnitium uenissent in aggresti scamno assidentem foco atque in catino ligneo cenantem magnumque auri pondus attulissent ac benignis uerbis inuitarent, ut auro uti uellet uultum risu soluit dicens : Superuacua ne dicam inepte aurum attulistis. Ite et dicite Samnitibus Ma. Curium male locupletibus imperare, quam eum fieri locupletem. At istud ut preciosum ita malo homini excogitatum munus refertote et mementote me neque in acie uinci neque pecunia posse corrumpi (fol. ccix d).

II.72.1 Ung checun : l'emploi absolu du pronom ung n'est pas attesté dans le texte, mais l'on trouve bien ung checun (I.45.1, I.46.2, I.59.6, I.85.6, I.110.1, I.113.5, I.119.1, I.147.3, etc.).

II.77.3-4 Et, pour ce, nous raconte Valere que ... : Senatus Fabricii Luscini Scipionisque filias ab indotatis nuptiis liberalitate sua uindicauit, quoniam paternae hereditati praeter opimam gloriam nihil erat quod acceptum referrent. (Val. Max. 4.4.10).

II.77.5-11 Pareillement, raconte ycelui Valere que ... : Curii et Fabricii Q. Tuberonem cognomine Catum discipulum fuisse merito quis existimauerit. Cui consulatum gerenti cum Aetolorum gens omnis usus uasa argentea magno pondere et exquisita arte fabricata per legatos misisset, qui superiore tempore gratulandi causa ad eum profecti retulerant fictilia se in eius mensa uidisse, monitos ne continentiae quasi paupertati succurrendum putarent cum suis sarcinis abire iussit. Quam bene Aetolicis domestica praetulerat, si frugalitatis eius exemplum posterior aetas sequi uoluisset ! Nunc quo uentum est ? A seruis impetrari uix potest ne eam supellectilem fastidiant, qua tunc consul uti non erubuit. (Val. Max. 4.3.7).

II.81-82 Les quelques ajouts d'Anjourrant pourraient correspondre à de simples développements des éléments implicites du texte, à moins, hypothèse peu probable, qu'il ait suivi la Vie d'Aratos de Plutarque. ${ }^{25}$

25 Dyck 1996, 474. 
II.88.2 $d u$ philosophe Panecius : emendation de $\mathrm{R}$ justifiée par la désignation de Panecius ailleurs dans le texte : «ce philozophe Panecius» (I.7.1, I.91, I.152.3, I.160.8, II.35.7, II.86.2).

III.16.5 les sept sages qui ont esté à Atheines ... : liste exemplaire des septs sages de Grèce ; voir, par exemple, Val. Max. 4.1.7.

III.38.1-10 Et, à ce propos, nous recite Platon ... : l'expansion de l'histoire de Gyges, roi de Lydie, pourrait sembler aller au-delà du développement des éléments implicites du texte. Pour autant, il est improbable qu'Anjourrant ait eu accès à la source de cette version du récit, à savoir La République de Platon (II, 359c). Par ailleurs, la version, plus accessible, de Justin (Just. Epit. 1.7) est différente de celle donnée ici.

III.41.1-4 Mais, il ne fut pas ainsi de Romulus ... : pour décrire le meurtre de Romulus par Rémus, il se peut qu'Anjourrant ait suivi Lucain (Ver. hist. 1.95) ou saint Augustin (De ciuitate Dei 15.5), et non la version, plus édulcorée, de Tite Live (1.7.2).

III.43.6-9 Car, comme j'ay dit en mon livre "de amitié » : développement provenant de Amic. 13.

III.45.1-7 Mais, je parle des communes amitiez ... : le développement de cet épisode pourrait s’appuyer sur Valère Maxime (Val. Max. 4.7.ext.1, où apparaît justement l'identification des deux amis comme des disciples de Pytagore), voire, moins probablement, de deux autres textes de Cicéron (Fin. 2.79 et Tusc. 5.63) ; sur les sources de cet épisode, voir Testard 1984, II, p. 93.

III.46.4 la principalle ville de Athaye ... : la leçon de R pourrait être difficilior ; l'on trouve chez Isidore de Séville : Corinthum in Achaia condidit Corinthus Orestis filius (Étymologies 15.1.45).

III.46.4-5 Mais, bien souvent, on peche ... : Cicéron avait déjà développé cette histoire au paragraphe I.35.

III.46.6-9 Semblablement, les Atheniens ... : le développement de ce passage du texte-source pourrait provenir de Valère Maxime (Val. Max. 9.2.ext.8).

III.47.5 à compaignie de pluseurs princes : émendation de $\mathrm{R}$ : il s'agirait de la seule graphie gne pour gnie dans le texte de R.

III.58.9 grant foison de poisson : émendation de la leçon de R (poison) : il s'agirait de la seule forme avec $s$ pour noter la sifflante sourde intervocalique ; la graphie poisson est d'ailleurs confirmée par les autres formes du mot dans le contexte (III.58.6 et III.59.1).

III.65.4 il est tenu de : une attestation d'estre tenu à au chapitre III.52.9. La structure pléonastique qui présente le complément du verbe sous forme pronominale et sous forme complète est également attestée en III.67.14 ; nous l'avons donc conservée. 
III.67.14 il fault que tu le entendes : pour une telle structure, voir III.65.4.

III.78.1 le pastour du roy de Lidie : épisode évoqué en III.38.

III.79 Aprés que Gayus Marius ... : développements probablement issus de Salluste (Iug. 64-65).

III.82.6 donna sa fille en mariage à Cesar : c'est Pompée qui épousa la fille de César et non l'inverse.

III.94.2-4 affin que nous retournions aux fables ... : source générale possible : Ov., Met. 2.1-332.

III.94.6-12 Semblablement, Theseus ... : histoire déjà développée en I.32.

III.97-98 Les développements sur Ulysse, Ajax et Thélémaque pourraient provenir d'Ovide (Met. 13, v. 1-398), éventuellement par l'intermédiaire de l'Ovide moralisé (De Boer 1915-1938, t. IV, v. 1304-1441).

III.99-100 L'amplification de l'exemplum de Marcus Atilius Regulus relève certes d'un processus de clarification et de narrativisation observable ailleurs dans la traduction (cf. Introduction, section 3.2-5). Cependant, il est possible qu'Anjourrant s'appuie sur des sources complémentaires, par exemple Tite Live (22.60.15), Valère Maxime (1.1.114; 2.9.8 ; 4.4.6), Frontin (Str. 2.3.10) ou Orose (Historiarum aduersum paganos 2.4.10). ${ }^{26}$

III.102.12 Actreus : Anjourrant confond ici l'auteur (Actius) et l'une de ses œuvres (l'Atrée).

III.109.1-9 Semblablement, Titus Vecturius et Spurius Postumus ... : le développement pourrait provenir de Valère Maxime (6.1.9), Tite Live (9.8.8) ou Orose (3.15.2).

III.109.11-16 Et, long temps aprés, Gayus Mantinus ... : le développement pourrait s’appuyer sur Valère Maxime (2.7.1) ou sur Cicéron (De republica 3.28).

III.112 Cet épisode apparaît dans le Breuiloquium de Jean de Galles, qui suit le De officiis de Cicéron (fol. ccII d) ; les quelques développements d'Anjourrant pourraient s'appuyer sur Tite Live (7.3.8-9 et 7.9) ou Valère Maxime (5.4.3).

III.113.5 il avoit oublié de faire quelque chose à Romme : contre-sens : le prisonnier dont il est question retourne au camp d'Hannibal, et non à Rome.

III.114.1-9 Mais, le senat fist bien encore plusgrant chose ... : les développements pourraient provenir de Tite Live (22.52.3, 22.58.4-5 et 22.59.9).

26 Sur cette question, voir aussi Dyck 1996, 622-623. 